\title{
On the Computation of a Minimum Phase Factor
}

This paper was downloaded from TechRxiv (https://www.techrxiv.org).

\section{LICENSE}

CC BY 4.0

SUBMISSION DATE / POSTED DATE

08-02-2022 / 10-02-2022

CITATION

Olivier, JC; Barnard, Etienne (2022): On the Computation of a Minimum Phase Factor. TechRxiv. Preprint. https://doi.org/10.36227/techrxiv.19134983.v1

$\mathrm{DOI}$

10.36227/techrxiv.19134983.v1 


\title{
On the Computation of a Minimum Phase Factor
}

\author{
J.C. Olivier and E. Barnard
}

\begin{abstract}
The accurate and efficient computation of a minimum phase factor for a high order linear phase finite impulse response system is considered. The paper shows that the residual errors achieved by current state of the art methods are nowhere near the smallest error possible on a finite resolution digital machine. This is shown to be due to conceptual errors in the literature pertaining to what constitutes a factorable system. This paper proves that factorization is only possible with a zero error (in the absence of machine finite resolution error) if the linear operator or matrix representing the linear phase filter $g$ is positive definite. This requirement is shown to be incompatible with the lifting method originally proposed in 1970, and that is still best practice at the time of writing. Methodology is proposed in this paper to mitigate these issues, and that is able to compute a minimum phase factor that is optimal - in the sense that errors are limited only by the finite precision of the digital computer, with no systematic error. The paper presents two practical applications of the proposed methodology: a) To compute a minimum phase Chebyshev finite impulse response filter, and b) Minimum phase system design that is unconditionally stable. Results are compared to state of the art methods from the literature and it is shown that the proposed methodology is able to reduce currently achievable residual errors by several orders of magnitude.
\end{abstract}

Index Terms-Minimum phase factorization, unitary matrix transformation, polynomial factorization.

\section{INTRODUCTION}

The theory and practice of minimum phase systems and their application have been studied for more than eight decades [1], [2], [3] and are well understood. The potential advantages offered by a minimum phase system depends on the application. For example the spectral response of a physical system may be known (measured) in terms of its magnitude only, and under such conditions a minimum phase transformation offers computational advantages [4]. For communications systems it is known that an optimal receiver must deploy a feedforward (anti-causal) filter after the optimal matched filter [5], [6]. In practice the minimum mean squared error (MMSE) estimator is often deployed to design the feedforward filter [7]. It is known that filter synthesis based on MMSE is approximate and does not guarantee a minimum phase impulse response, yet MMSE synthesis is widely deployed in practice. While it is true that modern communication systems based on orthogonal frequency domain multiplexing (OFDM) do not require a conventional matched filter-minimum phase receiver, there are applications where time domain signal processing is preferred [8]. As such this application of minimum phase transformation theory remains important and relevant.

Historically the computation of a minimum phase factor was developed on the $Z$ (spectral) domain. The computation

J.C. Olivier is with the School of Engineering, University of Tasmania, Sandy Bay Road, Hobart, Australia. E. Barnard is with Multilingual Speech Technologies (MuST), North-West University, South Africa. of a minimum phase factor based on computing the roots of a polynomial on the $Z$ domain is well understood [2], [3], [8]. In principle computing the roots of the polynomial provides a solution to the factorization problem, but that is practical only for low order polynomials. No new theoretical advances were made for the factorization of high order polynomials until 2003, when Orchard and Wilson showed that spectral factorization can be performed by solving a set of nonlinear equations [9], [10] — referred to in this paper as the O$\mathrm{W}$ equations, and no further theoretical advances have been proposed for factorization since that time.

The O-W equations have implications for the implementation of a minimum phase transformation on a digital computer. This was demonstrated by Kidambi and Antoniou in 2017 [11], and [11] presented results for the design of minimum phase finite impulse response (FIR) Chebyshev filters, reporting residual errors orders of magnitude smaller than those obtained through competing methods [12], [13], [14], [8]. In terms of computational requirements, the Hilbert transform and the cepstrum are both based on the discrete Fourier transform $(\mathrm{DFT})^{1}$ and require very long FFT's to obtain good FIR filter performance. On the other hand, [11] showed that solving the $\mathrm{O}-\mathrm{W}$ equations is very efficient. The methodology and results proposed in [11] have not been improved upon since its publication in 2017, and there are no methods available in the open literature able to yield a Chebyshev minimum phase filter with a smaller residual error than that reported in [11].

This paper analyses and develops the factorization problem based on a discrete time domain formulation, and analytical results based on linear algebra are derived. It will be demonstrated that the discrete time domain method is able to factorize the difficult case of the Chebyshev approximation based on matrix regularization. For spectral factorization this case was originally considered by Herrmann et al, based on the so-called "lifting" procedure [15]. Regularization is required because of the requirement of having a positive definite Hermitian matrix $\mathbf{G}$ representing the linear phase filter $\mathrm{g}$ on the discrete time domain, and will be shown to implicitly perform lifting through the matrix regularization. However there are subtle differences between the lifting procedure and matrix regularization, which will be demonstrated based on analytical results using a non-linear solver in the Mathematica software package. It will also be proved that in the limit where the matrix $\mathbf{G}$ is large (infinite), the $\mathrm{O}-\mathrm{W}$ equations are solved through Cholesky factorization of $\mathbf{G}$.

The discrete time domain formulation also yields a unitary matrix $\mathbf{F}$, that will be proved to be an all pass anti-causal filter. This matrix will be shown to represent the feedforward filter required for the design of an optimal discrete time receiver,

\footnotetext{
${ }^{1}$ Implemented through the fast Fourier transform (FFT).
} 
originally proposed in [5] for the continuous time case. The time domain formulation guarantees that the system is minimum phase, and a numerical example will be provided where MMSE design [7] fails, but where the proposed algorithm operates correctly.

The paper is structured as follows. In Section II factorization in the discrete time domain is proposed, based on locally Toeplitz matrices that are defined. Section III presents numerical results for the factorization of a 25 tap filter presented in [11], and it is demonstrated that based on 64 bit MATLAB software the Chebyshev minimum phase FIR filter residual error is several orders of magnitude smaller than the error reported in [11]. Section IV presents numerical results for the transformation of a given arbitrary phase FIR to a minimum phase FIR, and numerical results based on the proposed algorithm are compared to results obtained through MMSE design. The paper is concluded in Section V.

\section{Computing A Minimum Phase Factor}

\section{A. Locally Toeplitz matrices and symmetry point equilibrium}

In anticipation that Cholesky decomposition will be required to factorize on the time domain [7], this subsection proposes the deployment of locally Toeplitz matrices that operate on vectors with only local support near the symmetry point.

Consider the transpose of an upper triangular matrix obtained through Cholesky decomposition, as shown in Figure 1. The matrix is not strictly Toeplitz, as the top rows and the bottom rows differ from the rows near the symmetry point, where it is locally Toeplitz. This is typical when the matrix size is significantly larger than the number of diagonals that contain non-zero values. Such a matrix is exhibiting symmetry point equilibrium.

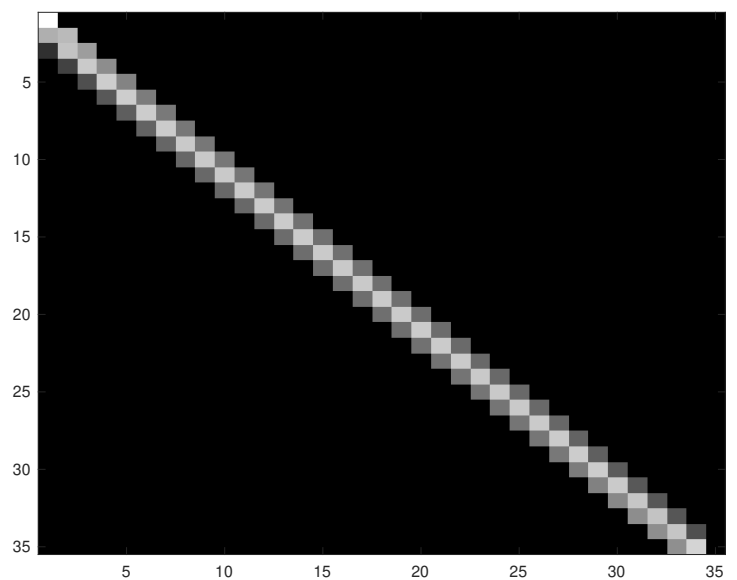

Figure 1. The structure of a matrix where symmetry point equilibrium holds — it is locally Toeplitz, but not strictly Toeplitz.

A matrix that is locally Toeplitz is able to represent a timeinvariant filter, provided it is operating on a vector that has only local support near the symmetry point. To make this statement clear, define an augmented FIR filter so that it has only local support, denoted as $\mathbf{h}_{\text {aug }}$ and defined $\mathbf{a s}^{2}$

$$
\mathbf{h}_{\text {aug }}=\{\underbrace{0, \cdots, 0}_{Q \text { zeros }}, h_{M-1}, \cdots, h_{1}, h_{0}, \underbrace{0, \cdots, 0}_{Q \text { zeros }}\}^{\mathrm{T}}
$$

where $Q \gg M$. As $\mathbf{h}_{\text {aug }}$ has support only near the symmetry point, a suitable locally Toeplitz matrix where symmetry point equilibrium holds is able to perform a time-invariant convolution operation on $\mathbf{h}_{\text {aug }}$, even if the matrix is not strictly Toeplitz.

To perform convolution, define a Toeplitz matrix with $(2 Q+M)$ columns and rows, given by

$\mathbf{H}=\left[\begin{array}{llllllll}h_{0} & h_{1} & \ldots & h_{M-1} & 0 & 0 & \cdots & 0 \\ 0 & h_{0} & h_{1} & \cdots & h_{M-1} & 0 & \cdots & 0 \\ \vdots & \vdots & \vdots & \vdots & \vdots & \vdots & \vdots & \vdots \\ 0 & \cdots & 0 & h_{0} & h_{1} & \cdots & \cdots & 0 \\ 0 & 0 & \cdots & 0 & h_{0} & h_{1} & \cdots & 0 \\ \vdots & \vdots & \vdots & \vdots & \vdots & \vdots & \vdots & \vdots \\ 0 & \cdots & \cdots & \cdots & \cdots & \cdots & 0 & h_{0}\end{array}\right]$.

Now define an augmented Kronecker delta as

$$
\delta_{\text {aug }}=\{\underbrace{0, \cdots, 0}_{Q \text { zeros }}, \underbrace{0, \cdots, 0}_{M-1 \text { zeros }}, 1, \underbrace{0, \cdots, 0}_{Q \text { zeros }}\}^{\mathrm{T}}
$$

then it follows that

$$
\mathbf{h}_{\text {aug }}=\mathbf{H} \delta_{\text {aug }} .
$$

Define the Gramian matrix $\mathbf{G}$ as

$$
\mathbf{G}=\mathbf{H}^{\mathrm{T}} \mathbf{H}
$$

then it follows that

$$
\mathbf{H}^{\mathrm{T}} \mathbf{h}_{\mathrm{aug}}=\mathbf{H}^{\mathrm{T}} \mathbf{H} \delta_{\mathrm{aug}}=\mathbf{G} \delta_{\mathrm{aug}} .
$$

$\mathbf{G}$ is symmetric, Toeplitz and Hermitian, and represents a linear phase FIR filter [16] denoted as g. The matrix $\mathbf{G}$ has causal non-zero diagonals (the main diagonal and upper triangular part), as well as non-zero diagonals that are anticausal (the lower triangular part). The dominant (centre) tap of $\mathrm{g}$ is represented by the main diagonal of $\mathbf{G}$.

\section{B. An approximate minimum phase factor $\mathbf{c}_{\text {approx }}$}

On the time domain the linear phase filter $\mathrm{g}$ and its matrix representation $\mathbf{G}$ will be factorable if $\mathbf{G}$ is also positive definite, as then Cholesky factorization can be performed to yield an upper triangular matrix $\mathbf{C}$ as [17]

$$
\mathbf{G}=\mathbf{C}^{\mathrm{T}} \mathbf{C} \text {. }
$$

It follows that

$$
\mathbf{H}^{\mathrm{T}} \mathbf{h}_{\text {aug }}=\mathbf{C}^{\mathrm{T}} \mathbf{C} \delta_{\text {aug }} \Longrightarrow \underbrace{\left[\left(\mathbf{C}^{\mathrm{T}}\right)^{-1} \mathbf{H}^{\mathrm{T}}\right]}_{\text {Matrix } \mathbf{F}} \mathbf{h}_{\text {aug }}=\mathbf{C} \delta_{\text {aug }} .
$$

${ }^{2}$ The fact that the vector has time $n$ advancing from right to left is in anticipation of the fact that Cholesky factorisation implemented by most computing platforms provides a factorisation in the form $\mathbf{U}^{T} \mathbf{U}$ where $\mathbf{U}$ is upper triangular 
The matrices $\mathbf{C}$ and $\mathbf{F}$ are locally Toeplitz (if $Q$ is sufficiently large), and since $\mathbf{h}_{\text {aug }}$ and $\delta_{\text {aug }}$ have only local support, these matrices represent time-invariant filters.

Thus for a sufficiently large value of $Q$ it follows that

$$
\mathbf{c}_{\text {aug }}=\mathbf{C} \delta_{\text {aug }}=\mathbf{F} \mathbf{h}_{\text {aug }}
$$

where $\mathbf{c}_{\text {aug }}$ is given by

$$
\mathbf{c}_{\text {aug }}=\{\underbrace{0, \cdots, 0}_{Q \text { zeros }}, c_{M-1}, \cdots, c_{1}, c_{0}, \underbrace{0, \cdots, 0}_{Q \text { zeros }}\}^{\mathrm{T}} .
$$

Cholesky decomposition expresses a Hermitian positive definite matrix as the product of a minimum phase matrix and its match [17], regardless of the value of $Q$. Thus when symmetry point equilibrium holds, it follows that a minimum phase factor can be recovered from $\mathbf{c}_{\text {aug }}$ as

$$
\mathbf{c}_{\text {approx }}=\left\{c_{0}, c_{1}, c_{2}, \cdots, c_{M-1}\right\}^{\mathrm{T}} .
$$

$\mathbf{c}_{\text {approx }}$ is approximate as $Q$ is finite, but it will be shown in Section III that $\mathbf{c}_{\text {approx }}$ is remarkably accurate, even for moderate settings of $Q$. Section III will also demonstrate that $\mathbf{c}_{\text {approx }}$ is an appropriate choice as an initial guess to perform numerical optimization of the non-linear $\mathrm{O}-\mathrm{W}$ equations to be derived below. In Subsection II-C it will be formally shown that if $Q \rightarrow \infty$ then $\mathbf{c}_{\text {approx }} \rightarrow \mathbf{c}$, where $\mathbf{c}$ is the optimal minimum phase factor.

\section{A minimum phase factor $\mathbf{c}$ in the limit where $Q \rightarrow \infty$}

With $Q$ set to a sufficiently large but finite value, an approximate factor $\mathbf{c}_{\text {approx }}$ is obtained as shown in the previous subsection. This section shows that if the limit is taken and $Q \rightarrow \infty$, then an exact solution is obtained through a set of non-linear equations. To solve these equations based on numerical optimization, $\mathbf{c}_{\text {approx }}$ serves as an accurate starting point for fast convergence and accurate results.

When $Q \rightarrow \infty$ symmetry point equilibrium clearly holds, and $\mathbf{C}$ is locally Toeplitz near the symmetry point. The matrix is factorized as

$$
\mathbf{G}=\mathbf{C}^{\mathrm{T}} \mathbf{C}
$$

so that for any row near the symmetry point of $\mathbf{C}^{\mathrm{T}}$, say row $j$, multiplied with columns $j, j+1, j+2, \cdots$ of $\mathbf{C}$ yields a system of non-linear equations given by

$$
\begin{aligned}
c_{0}^{2}+c_{1}^{2}+\cdots+c_{M-1}^{2} & =g[M-1] \\
c_{0} c_{1}+c_{1} c_{2}+\cdots+c_{M-2} c_{M-1} & =g[M-2] \\
c_{0} c_{2}+c_{1} c_{3}+\cdots+c_{M-3} c_{M-1} & =g[M-3] \\
\vdots & =\vdots \\
c_{0} c_{M-1} & =g[0] .
\end{aligned}
$$

This system of non-linear equations is identical to the O-W equations proposed in [9] where spectral factorization was deployed. Hence in the limit where $Q$ is infinite, time domain factorization is identical to spectral factorization, a known result from linear systems theory [16].
Table I

A LOW ORDER LINEAR PHASE FILTER $\mathbf{g}$

\begin{tabular}{cc}
\hline \hline Tap number $n$ & $\mathbf{g}$ \\
\hline 0 & 0.066075742625345 \\
1 & 0.239064282650394 \\
2 & 0.347182106755652 \\
3 & 0.239064282650394 \\
4 & 0.066075742625345 \\
\hline
\end{tabular}

Define the residual error $\mathbf{e}$ of the non-linear equations above as

$$
\begin{aligned}
c_{0}^{2}+c_{1}^{2}+\cdots+c_{M-1}^{2}-g[M-1] & =e_{0} \\
c_{0} c_{1}+c_{1} c_{2}+\cdots+c_{M-2} c_{M-1}-g[M-2] & =e_{1} \\
c_{0} c_{2}+c_{1} c_{3}+\cdots+c_{M-3} c_{M-1}-g[M-3] & =e_{2} \\
\vdots & =\vdots \\
c_{0} c_{M-1}-g[0] & =e_{M-1} .
\end{aligned}
$$

Denote the norm of $\mathbf{e}$ as $\mathrm{E}_{\mathrm{L}_{2}}$. It will be demonstrated below that the norm plays a fundamental role in factorization.

In Appendix $\mathbf{A}$ it is proved that $\mathbf{F}$ is unitary, and an all pass filter. From equation (9) it is known that

$$
\mathbf{F h}_{\text {aug }}=\mathbf{C} \delta_{\text {aug }} \triangleq \mathbf{c}_{\text {aug }} .
$$

$\mathbf{F}$ is an all pass filter, hence it follows that

$$
|C(\Omega)|=|H(\Omega)| .
$$

In Section IV the salient properties of the matrix $\mathbf{F}$ will be further explored.

\section{Factorizing $\mathbf{G}$ and Regularization}

The Hermitian matrix $\mathbf{G}$ has real eigenvalues [17], and as remarked above will be factorable based on Cholesky factorization if $\mathbf{G}$ is positive definite. Thus in order to be factorable, the minimum eigenvalue of matrix $\mathbf{G}$ must satisfy

$$
\lambda_{\min }>0 \text {. }
$$

Depending on the application $\mathbf{G}$ may well be positive definite, but for certain applications $\mathbf{G}$ is not positive definite ${ }^{3}$, and the minimum eigenvalue will be negative. To mitigate this problem and to guarantee that the Gramian is factorable, the main diagonal can be modified, with the modified matrix denoted as $\mathbf{G}_{\mathrm{adj}}$ and given by

$$
\mathbf{G}_{\mathrm{adj}}=\mathbf{G}+\gamma \mathbf{I}
$$

$\mathbf{G}_{\text {adj }}$ is guaranteed to be factorable as

$$
\mathbf{G}_{\text {adj }}=\mathbf{C}^{\mathrm{T}} \mathbf{C}
$$

if, and only if, $\gamma$ is chosen as

$$
\gamma>\left|\lambda_{\min }\right|
$$

as then the smallest eigenvalue is finite and positive ${ }^{4}$ [17], [18].

\footnotetext{
${ }^{3}$ This is the case when a Chebyshev linear phase filter is factorized.

${ }^{4}$ Known as regularization.
} 


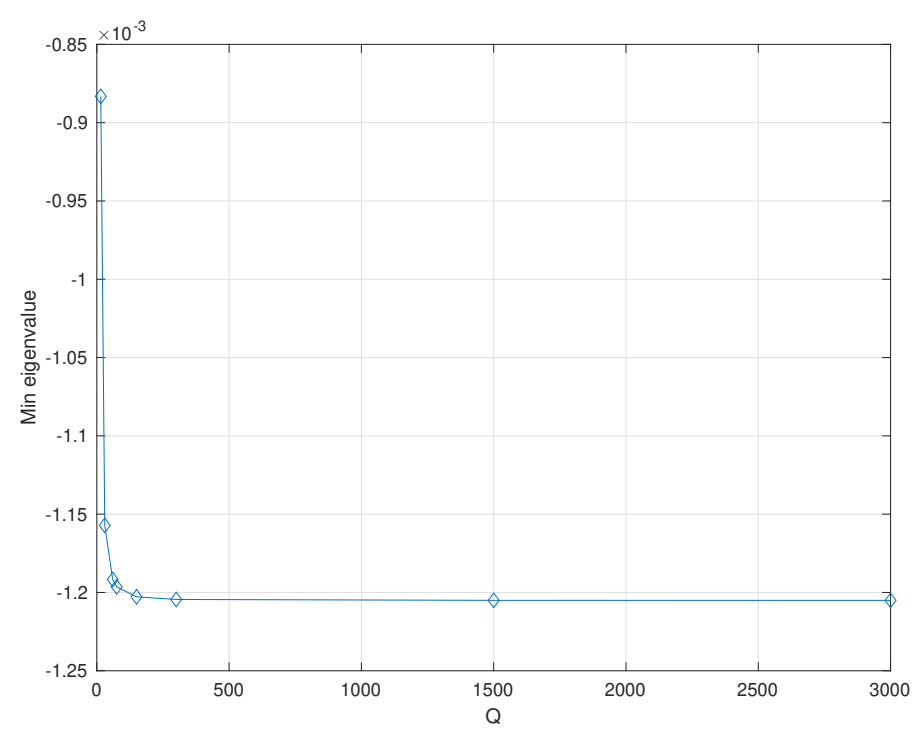

Figure 2. The value of the minimum eigenvalue as a function of $Q$.

To demonstrate the relation and the subtle differences between regularization and lifting (proposed by Herrmann [15]), consider a low order linear phase filter $\mathbf{g}$ shown in Table I. The lifting value is denoted as $\tau$, and for this filter is given by

$$
\tau=0.00120505352635236 \text {. }
$$

If adding $\tau$ to the dominant tap of $\mathrm{g}$ yields a factorable filter, then an analytical solution in the real number field for the $\mathrm{O}$ $\mathrm{W}$ equations must be possible, and will yield a residual error of zero. An anlytical solution can be obtained by making use of Mathematica, the outcome of which is shown in Figure 3 . It is clear that the lifting procedure does not yield a real solution for the $\mathrm{O}-\mathrm{W}$ equations. All the solutions provided by Mathematica are complex. Any attempt to solve the O-W equations with the factor $\mathrm{c}$ real (as it should be), will naturally lead to $\mathrm{E}_{L_{2}}>0$. Hence lifting as proposed by Herrmann [15] does not provide a factorable linear phase filter, if factorable is defined as the requirement to have the $\mathrm{O}-\mathrm{W}$ equations yield a real solution with $\mathrm{E}_{L_{2}}=0$ (in the absence of machine error). This observation leads to a revised definition of factorable that is proposed in this paper:

Definition 1. A linear phase filter $\mathbf{g}$ is factorable, if and only if, $\mathrm{E}_{L_{2}}=0$ and the factor $\mathbf{c}$ is real.

It will now be demonstrated how a factorable $\mathrm{g}$ can be constructed based on regularization. The minimum eigenvalue of $\mathbf{G}$ is shown in Figure 2 as a function of $Q$. It is clear that as $Q$ is chosen to be much greater than $M$, the eigenvalue is converging to the lifting value $\tau$. Thus it is not required to compute the minimum eigenvalue of $\mathbf{G}$ for an infinite (very large) $Q$, as

$$
\left|\lambda_{\min }\right|=\tau \text { if } Q \rightarrow \infty
$$

But crucially regularization given by (20) demands that the value $\gamma$ added to the dominant tap must exceed the absolute

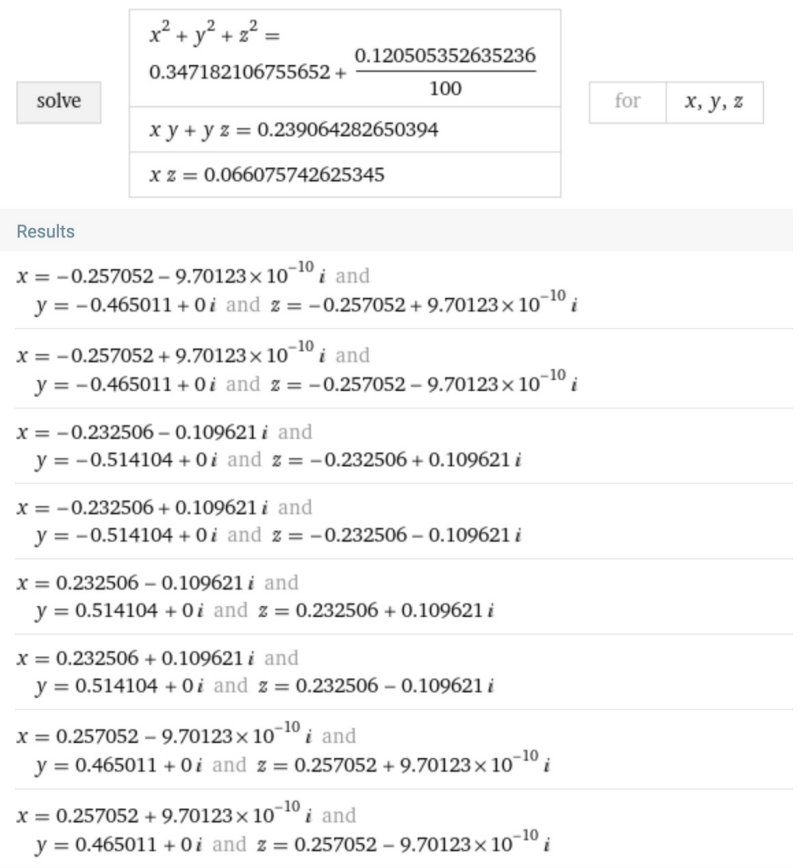

Figure 3. The solution provided by Mathematica for the non-linear Orchard and Wilson equations, with the dominant tap of $\mathbf{g}$ adjusted based on lifting. Clearly a real solution does not exist.

value of the minimum eigenvalue. Hence by adding a small number denoted by $\epsilon$ to $\left|\lambda_{\min }\right|$, say

$$
\gamma=\left|\lambda_{\min }\right|+3 \times 10^{-17}=\tau+3 \times 10^{-17}
$$

then (20) is satisfied, and as shown in Figure 4 the O-W equations can now be solved to yield a real solution with zero error (in the absence of finite machine resolution).

Unfortunately there is no known method for computing the number $\epsilon$, and in the next section a plot of $\mathrm{E}_{L_{2}}$ as a function of $\gamma$ is proposed as an effective strategy to determine $\epsilon$.

Results based on the exact solution are of course theoretical, and if a numerical solution is performed on a digital computer with finite resolution, then $\mathrm{E}_{L_{2}}>0$. But what must be realised is that this error is induced by finite machine resolution, and can be reduced simply by improving the computer resolution.

Section III will provide numerical results based on a practical 25 tap filter to verify the conclusions reached in this section, and will compare the results to state of the art methodology available in the literature.

\section{Application 1: A Chebyshev Minimum PHASE FACTOR}

A linear phase filter $\mathbf{g}$ presented as example 1 in [11] is deployed in this section. The 25 tap settings for vector $\mathbf{g}$ is shown in Table II. The frequency response and the pole/zero representation of $\mathbf{g}$ are shown in Figure 5. It is clear that g cannot be factored - and to confirm this, note that the minimum eigenvalue of the Gramian matrix is negative. 


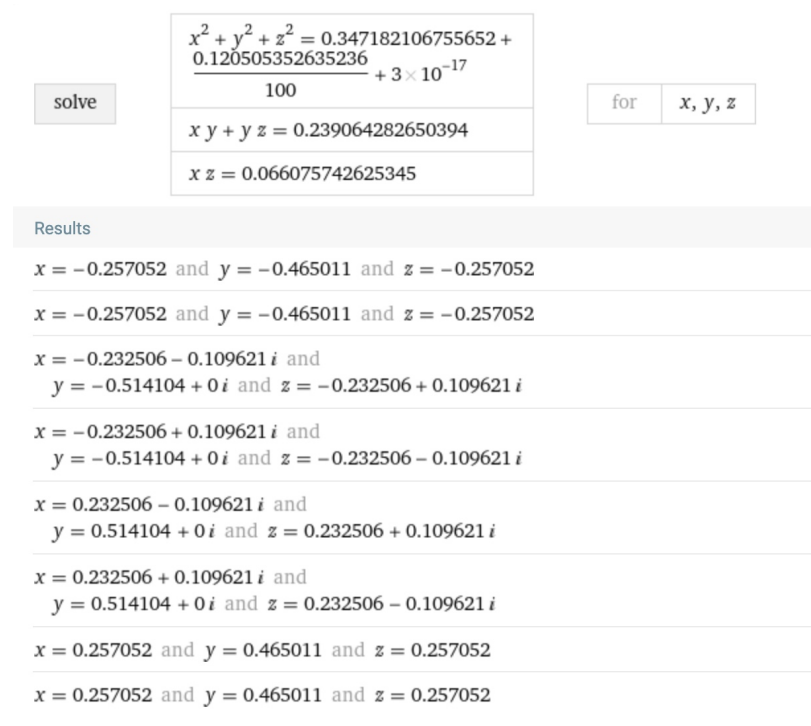

Figure 4. The solution provided by Mathematica for the non-linear Orchard and Wilson equations, with the dominant tap of $\mathbf{g}$ adjusted based on regularization with $\mathrm{g}$ positive definite. In this case there is a real solution.

Table II

TAP SETTINGS FOR g FROM [11]

\begin{tabular}{cc}
\hline \hline Tap number $n$ & $\mathbf{g}$ \\
\hline 0 & -0.00033409853951949 \\
1 & -0.002489549410806 \\
2 & -0.007656350824928 \\
3 & -0.011354989160955 \\
4 & -0.002981767473881 \\
5 & 0.018180581093311 \\
6 & 0.026333770707396 \\
7 & -0.008295888670961 \\
8 & -0.062043244763120 \\
9 & -0.047371546549295 \\
10 & 0.095349066618093 \\
11 & 0.295504051520742 \\
12 & 0.391016383693520 \\
13 & 0.295504051520742 \\
14 & 0.095349066618093 \\
15 & -0.047371546549295 \\
16 & -0.062043244763120 \\
17 & -0.008295888670961 \\
18 & 0.026333770707396 \\
19 & 0.018180581093311 \\
20 & -0.002981767473881 \\
21 & -0.011354989160955 \\
22 & -0.007656350824928 \\
23 & -0.002489549410806 \\
24 & -0.00033409853951949 \\
\hline & \\
& \\
\hline &
\end{tabular}

A. Applying regularization to obtain a linear phase filter $\mathrm{g}$ that is factorable

To mitigate the non-factorability of the linear phase filter g, regularization is deployed. In this case the minimum eigenvalue was measured by examining the negative ripple peaks in $G(\Omega)$. The most negative peak yielded the minimum eigenvalue for $Q \rightarrow \infty$ as

$$
\left|\lambda_{\min }\right|=\tau=5.832240436431935 \times 10^{-6} .
$$

Now the residual error $E_{L_{2}}$ is computed by solving the nonlinear equations (13) through computer optimization. In this paper a 64 bit version of the Levenberg-Marquardt optimizer available in MATLAB as lsqnonlin was deployed, and included the derivatives (Hessian) as proposed in [11]. The initial guess
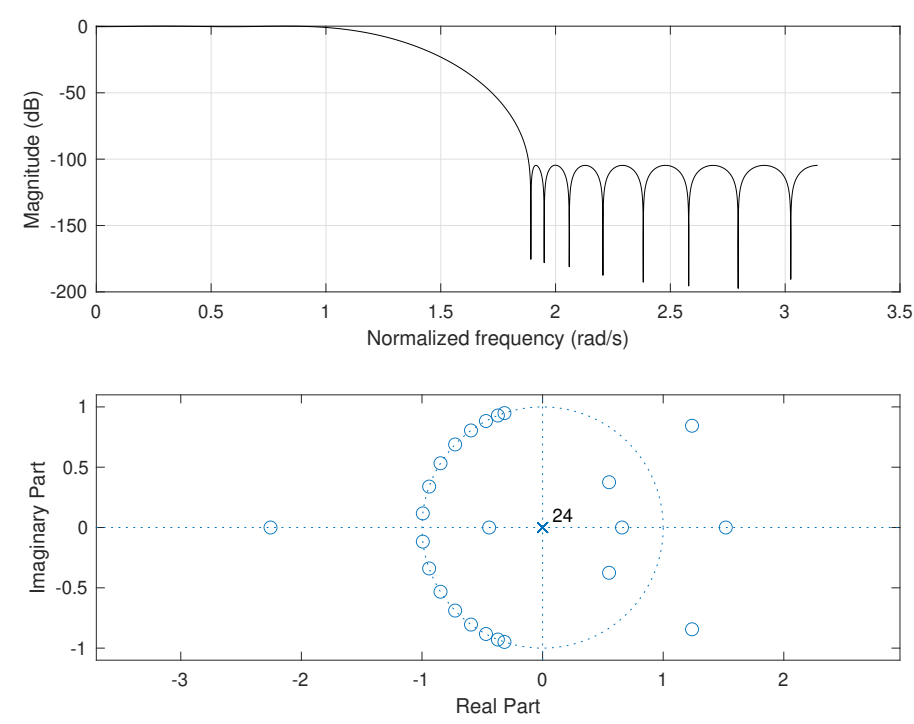

Figure 5. A lowpass filter $\mathbf{g}$ to be factorized in order to compute the minimum phase factor $\mathbf{c}$.

for c was computed on the time domain using Cholesky decomposition, with $Q=10 N$ and $N=25$ in this case (number of taps in $\mathbf{g}$ ).

The residual error $E_{L_{2}}$ as a function of $\gamma=\left|\lambda_{\min }\right|+\epsilon$ is shown in Figure 6, and the existence of a waterfall point where the error falls away is evident - this is where the value of $\gamma$ is exceeded by $\epsilon \approx 10^{-13}$, rendering $\mathrm{g}$ factorable and positive definite. This result confirms the time domain results where it was proved that the value of $\gamma$ must exceed the absolute value of the minimum eigenvalue (lifting value).

After the waterfall point, the residual error is limited by the digital machine resolution, and increasing $\gamma$ beyond this value would simply increase the stop-band leakage, and is thus counterproductive. Hence a factorable linear phase filter $\mathbf{g}$ can be computed by adding

$$
\gamma=\left|\lambda_{\min }\right|+1.16 \times 10^{-13}
$$

to the dominant tap, so that $\mathbf{g}$ is positive definite as required by regularization.

Note that the residual error reported by [11] (see Figure 6) is 4 orders of magnitude above the 64 bit machine resolution lower limit of $2.8 \times 10^{-17}$. Clearly the algorithm proposed and applied in [11] is insufficient to guarantee that $\mathbf{g}$ is factorable, hence leading to results that are not optimal. In spite of this conclusion, the results reported in [11] are state of the art at the time of writing.

The optimum minimum phase factor (FIR filter) $\mathbf{c}$ is shown in Table III. The tap settings for $\mathbf{c}$ differ in the fifth decimal place when compared to the values reported in [11]. In practice changes in the fifth decimal place of the taps of a FIR filter would be deemed significant, and clearly it is important to obtain a positive definite and factorable linear phase FIR filter.

The residual error is shown in Table IV for a number of methods available in the literature [11], and compared to the residual error based on a positive definite and factorable $\mathrm{g}$ as proposed in this paper. The method proposed in this paper 


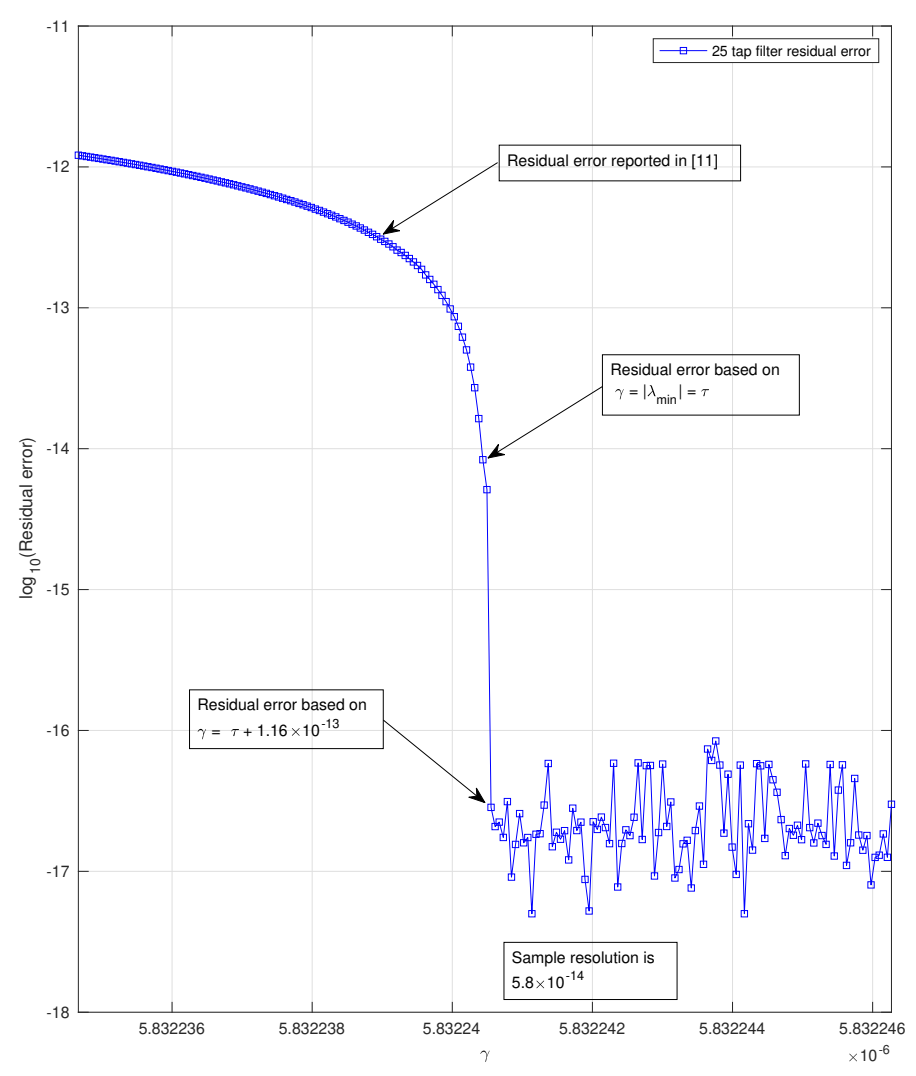

Figure 6. The residual error $E_{L_{2}}$ as a function of $\gamma$. Note the waterfall point, which occurs as soon as $\mathbf{g}$ becomes positive definite as required by regularization.

Table III

SETTINGS FOR THE APPROXIMATE $\mathbf{c}_{\text {approx }}$ AND THE OPTIMAL TAP SETTINGS FOR c.

\begin{tabular}{cccc}
\hline \hline Tap $n$ & $\mathbf{c}$ & $\mathbf{c}_{\text {approx }}(Q=10 N)$ & $\mathbf{c}$ from [11] \\
\hline 0 & 0.051115654818476 & 0.053170658603589 & 0.05111407271 \\
1 & 0.200710190492617 & 0.206160489181734 & 0.2007050616 \\
2 & 0.373675662335455 & 0.378228787870903 & 0.3736680124 \\
3 & 0.383763292854902 & 0.380981033331147 & 0.3837574877 \\
4 & 0.168110995957186 & 0.159498759291175 & 0.1681105336 \\
5 & -0.081211240469989 & -0.086362886469131 & -0.0812083306 \\
6 & -0.139792118109026 & -0.137267954224048 & -0.1397903352 \\
7 & -0.028413408959431 & -0.024127759326649 & -0.0284408539 \\
8 & 0.060844647358445 & 0.061160436307306 & 0.06084337463 \\
9 & 0.040660368538867 & 0.038606283413641 & 0.04066023143 \\
10 & -0.011537810825624 & -0.012290539783330 & -0.01153731949 \\
11 & -0.023042542956496 & -0.022479612134730 & -0.02304231216 \\
12 & -0.006536978978309 & -0.006283513281458 & -0.006536952812 \\
\hline
\end{tabular}

outperforms the current state or the art method available in [11] by four orders of magnitude.

Table IV

RESIDUAL ERROR NORM FOR VARIOUS METHODS AND THE PROPOSED POSITIVE DEFINITE $g$

\begin{tabular}{ccccc}
\hline \hline Proposed & From [11] & Cepstrum & DHT & Orchard [9] \\
\hline $2.8 \times 10^{-17}$ & $3.4 \times 10^{-13}$ & $2.4 \times 10^{-6}$ & $2.8 \times 10^{-10}$ & $3.4 \times 10^{-10}$ \\
\hline
\end{tabular}

\section{Application 2: An OPTIMUM Minimum PHASE RECEIVER AND FEEDFORWARD FILTER}

In this section factorization is applied to perform a minimum phase transformation on a known finite impulse response, and the anti-causal feedforward filter required to implement an optimal receiver [6] is computed. Results are compared to a transformation based on the MMSE estimator which is widely deployed in practice [7], [6]. The system is assumed to operate in additive white Gaussian noise, thus a noise whitening filter need not be deployed.

\section{A. MMSE estimator deployed to perform the minimum phase transformation}

A model valid for a system with a measured (or provided) FIR $\mathbf{h}$ is given by

$$
y[n]=\sum_{k=0}^{M} h[k] s[n-k]+w_{s}[n] .
$$

y represents an observed but noisy version of the system output in response to the system input $\mathbf{s}$. The noise samples $w_{s}[n]$ are assumed to be drawn from an independently and identically distributed Gaussian random process with variance $\sigma^{2}$ and zero mean.

The transformation of $\mathbf{h}$ to a minimum phase $\mathbf{c}$ can be performed by a feedforward (anti-causal) filter $\mathbf{f}$ [5], [6]. That is, the transformed system model is given by

$$
\sum_{j=0}^{P} f[j] y[n+j]=\sum_{k=0}^{M} c[k] s[n-k]+w_{f}[n] .
$$

The MMSE estimator computes filters $\mathbf{f}$ and $\mathbf{c}$ based on the orthogonality principle [7]. These ideas are rooted in the seminal work of Wiener and Kolmogorov, and the literature on this methodology is mature and complete [19], [20], [7]. In this section the MMSE estimator is applied to compute $\mathbf{c}$, and used as a benchmark to assess factorization applied to the transformation problem.

\section{B. Numerical results}

The example chosen is a random 10 tap FIR as shown in Figure 7 (top left). Figure 7 shows the results for transformation based on factorization, as well as based on the MMSE estimator. Note that the matched filter output on which factorization is based, is denoted by $\mathbf{g}$ and is a linear phase FIR filter. It is factorable with $\gamma=0$.

The MMSE estimator yielded the filter $\mathbf{c}$ as shown on the right in Figure 7. The frequency magnitude plot for the filter based on MMSE shows that there are frequencies where the magnitude response is not identical to that of $H(\Omega)$. For the proposed transformation the magnitude response is nearly identical to $H(\Omega)$ as expected.

What is concerning is that the MMSE estimator yielded a filter $\mathbf{c}$ that has a pair of zeros marginally outside the unit circle. In practice where feedback may be applied this would mean that the filter $\mathbf{c}$ is not unconditionally stable. In contrast factorization yields the correct placement for this zero pair, as shown in Figure 7. 

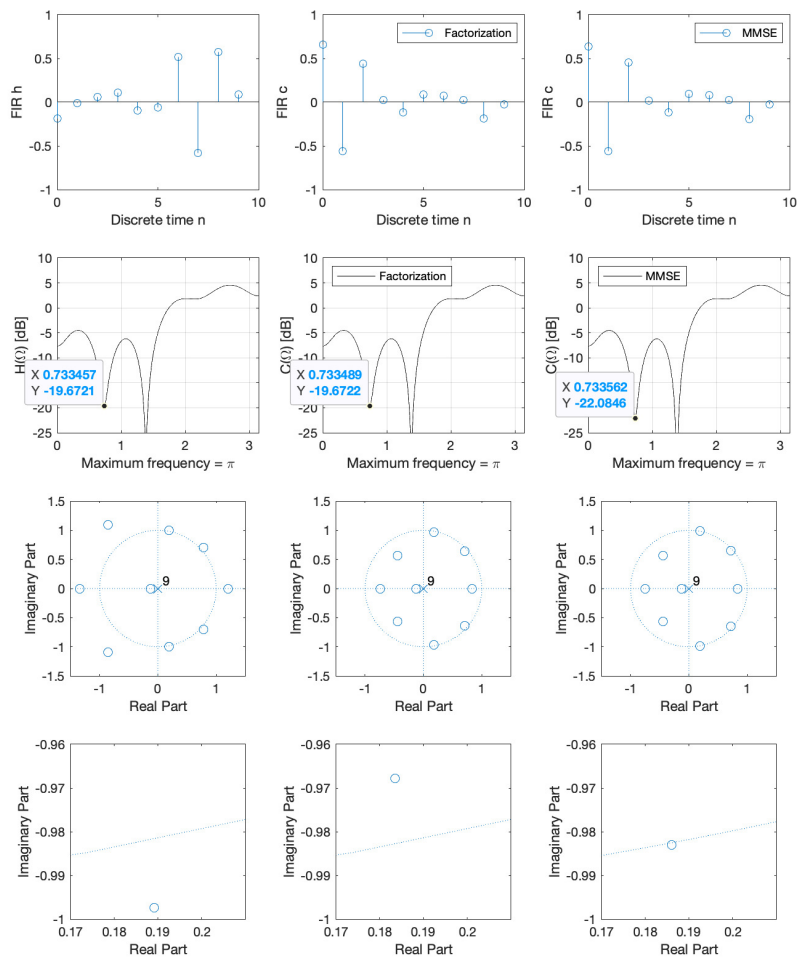

Figure 7. A 10 tap arbitrary phase FIR h, and the minimum phase FIR c based on factorization and the MMSE method [7].

Table V

RESIDUAL ERROR NORM FOR MMSE AND THE PROPOSED METHOD

\begin{tabular}{cc}
\hline \hline Proposed & MMSE [7] \\
\hline $6.11 \times 10^{-17}$ & $2.07 \times 10^{-3}$
\end{tabular}

The MMSE estimator does not provide a residual error that is anywhere near the machine resolution floor, as shown in Table V. Evidently an error on the order of $10^{-3}$ provides a filter $\mathbf{c}$ where $C(\Omega)$ is visibly sub-optimal as shown in Figure 7.

It is interesting to consider how factorization is able to obtain these results. Factorization yielded small but critical changes in the filter $\mathbf{c}$ when compared to MMSE, and these changes were able to guarantee that $\mathbf{c}$ is minimum phase. The difference comes down to the feedforward (anti-casual) filter $\mathrm{f}$, as shown in Figure 8. For factorization, the feedforward filter is represented by the matrix $\mathbf{F}$, which was demonstrated to be unitary and an all pass filter. Factorization computes the matrix $\mathbf{F}$ based on Cholesky decomposition, not based on the orthogonality principle as is the case for the MMSE estimator. The rows of matrix $\mathbf{F}$ (near the symmetry point where it is locally Toeplitz) contain $\mathbf{f}$, and is accurate due to the robust Cholesky decomposition.
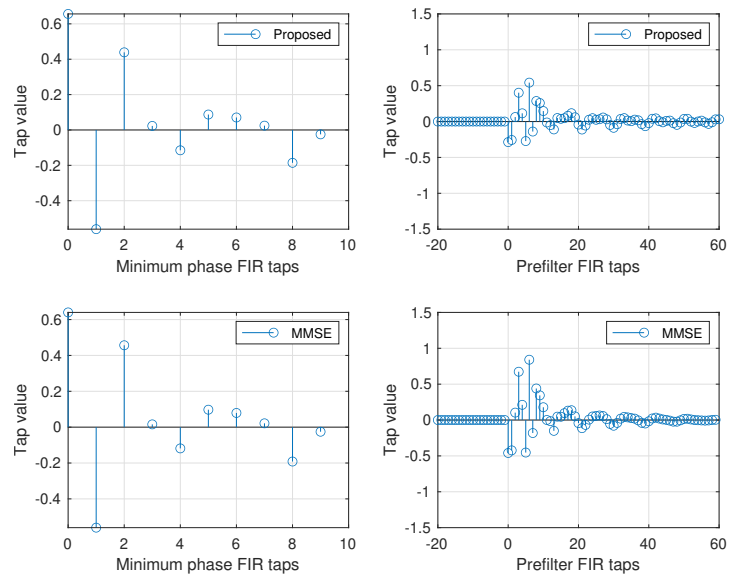

Figure 8. The anti-causal transformation filter $\mathbf{f}$ able to transform the given FIR $\mathbf{h}$ to a minimum phase filter $\mathbf{c}$.

Finally, note that matrix $\mathbf{F}$ is lower triangular ${ }^{5}$, that is, the transformation filter is purely anti-causal.

\section{CONCLusions}

The paper demonstrated that if the adjustment (or lifting) factor is set to the ripple peak, as proposed in [15], it does not produce a factorable equivalent for a linear-phase Chebyshev filter, and is in practice insufficient for that goal. The paper demonstrated that the definition of what constitutes a factorable linear phase filter has to be modified: it was proposed that the non-linear equations proposed in [9] be exactly solvable (up to machine precision error) by a real minimum phase filter, before a linear phase filter is deemed factorable.

The paper showed that this definition of factorability in practice demands that the linear phase filter be positive definite, not positive semi-definite as proposed by [15]. Based on a time-domain factorization of the given system, it was shown that the proposed definition for a factorable linear phase filter is correct, and that the non-linear equations proposed in [9] are exactly solvable if the linear phase filter is positive definite.

The consequences of a change from a positive semi-definite $\mathrm{g}$ to positive definite $\mathrm{g}$ are dramatic: when the residual error is plotted as a function of the dominant tap adjustment $\gamma$, it exhibits a waterfall point coinciding with the point where $\gamma$ guarantees factorability. At that point the residual error falls away to an error floor set by the finite resolution of the digital computer.

Time-domain factorization was shown to be possible if an augmentation of the impulse response is defined so that it has only local support, and becomes exact as the expansion approaches infinity. However, even for relatively small expansion factors useful approximations for the minimum phase factor are obtained. This served to provide a good initial starting point for optimization of the non-linear equations proposed in [9].

\footnotetext{
${ }^{5}$ Because the definition of the direction of time $n$ was reversed to conform to the upper triangular $\mathbf{C}$, a lower triangular matrix is anti-causal in this paper. Most Cholesky decomposition functions in commercial software yield an upper triangular matrix, for historical reasons.
} 
Numerical results were provided indicating that the proposed factorization yields a significant improvement in the residual error when compared to best practice available in the literature. Specifically it outperformed the results for the state-of-the-art methodology proposed in [11] by four orders of magnitude.

\section{REFERENCES}

[1] H. W. Bode, "Relations between attenuation and phase in feedback amplifier design," Bell System Technical Journal, pp. 421-454, 1940.

[2] A. V. Oppenheim and R. W. Schafer, Digital Signal Processing. Pearson Education, 2015.

[3] _ Discrete-Time Signal Processing. Prentice-Hall, 1989.

[4] R. Halir, I. Molina-Fernández, J. Wangüemert-Pérez, A. Ortega-Moñux, J. de Oliva-Rubio, and P. Cheben, "Characterization of integrated photonic devices with minimum phase technique," Optics express, vol. 17, pp. 8349-61, 062009.

[5] J. Salz, "Optimum mean-square decision feedback equalization," The Bell System Technical Journal, vol. 52, no. 8, pp. 1341-1373, 1973.

[6] J. G. Proakis and M. Salehi, Digital Communications. Boston: McGraw-Hill, 2008.

[7] N. Al-Dhahir and J. Cioffi, "MMSE decision-feedback equalizers: finitelength results," IEEE Transactions on Information Theory, vol. 41, no. 4, pp. $961-975,1995$.

[8] J. O. Smith, Introduction to Digital Filters with Audio Applications. [Online]. Available: https://ccrma.stanford.edu/jos/

[9] H. J. Orchard and A. N. Wilson, "On the computation of a minimum phase spectral factor," IEEE Trans. Circuits Syst. I, Fundam. Theory Appl., vol. 50, pp. 365-375, 2003.

[10] A. N. Wilson and H. J. Orchard, "On the computation of a minimum phase spectral factor," Linear Algebra and its Applications, vol. 411, p. 309, 2005.

[11] S. Kidambi and A. Antoniou, "Design of minimum-phase filters using optimization," IEEE Trans. Circuits and Systems - II: Express briefs, vol. 64, pp. 472-476, 2017

[12] N. D. Venkata, B. L. Evans, and S. R. McCaslin, "Design of optimal minimum-phase digital fir filters using discrete hilbert transforms," IEEE Trans. Signal Process., vol. 48, pp. 1491-1495, 2000.

[13] Y. Kamp and C. J. Wellekens, "Optimal design of minimum-phase fir filters," IEEE Trans. Acoust., Speech, Signal Process., vol. 31, pp. 922 926, 1983.

[14] G. R. Reddy, "Design of minimum-phase fir digital filter through cepstrum," Electron. Lett., vol. 22, pp. 1225-1227, 1986.

[15] O. Herrmann and H. W. Schuessler, "Design of non-recursive digital filters with minimum phase," Electron. Lett., vol. 6, pp. 329-330, 1970.

[16] T. Kailath, Linear Systems. Prentice-Hall, 1980.

[17] G. H. Golub and C. F. Van Loan, Matrix Computations, 2013.

[18] "Regularization," https://math.stackexchange.com/questions/3596910/regularizationof-a-matrix-using-a-diagonal-matrix, accessed: 2021-09-21.

[19] K. Vastola and H. Poor, "Robust wiener-kolmogorov theory," IEEE Transactions on Information Theory, vol. 30, no. 2, pp. 316-327, 1984

[20] T. Kailath, A. H. Sayed, and B. Hassibi, Linear Estimation. Upper Saddle River, N.J., Prentice Hall, 2000.

\section{APPENDIX A}

Theorem 1. Transformation matrix $\mathbf{F}$ is unitary

Proof. The proof starts by computing

$$
\mathbf{F} \mathbf{F}^{\mathrm{T}}=\left(\left(\mathbf{C}^{\mathrm{T}}\right)^{-\mathbf{1}} \mathbf{H}^{\mathrm{T}}\right)\left(\left(\mathbf{C}^{\mathrm{T}}\right)^{-\mathbf{1}} \mathbf{H}^{\mathrm{T}}\right)^{\mathrm{T}}
$$

and then demonstrates that this operation yields an identity matrix I. Proceeding in a step by step manner, it follows that

$$
\begin{array}{r}
\left(\left(\mathbf{C}^{\mathrm{T}}\right)^{-\mathbf{1}} \mathbf{H}^{\mathrm{T}}\right)\left(\left(\mathbf{C}^{\mathrm{T}}\right)^{-\mathbf{1}} \mathbf{H}^{\mathrm{T}}\right)^{\mathrm{T}} \\
=\left(\left(\mathbf{C}^{\mathrm{T}}\right)^{-\mathbf{1}} \mathbf{H}^{\mathrm{T}}\right)\left(\left(\mathbf{H}^{\mathrm{T}}\right)^{\mathrm{T}}\left[\left(\mathbf{C}^{\mathrm{T}}\right)^{-\mathbf{1}}\right]^{\mathrm{T}}\right) \\
=\left(\left(\mathbf{C}^{\mathrm{T}}\right)^{-\mathbf{1}} \mathbf{H}^{\mathrm{T}}\right)\left(\mathbf{H}\left[\left(\mathbf{C}^{\mathrm{T}}\right)^{-\mathbf{1}}\right]^{\mathrm{T}}\right) \\
=\left(\left(\mathbf{C}^{\mathrm{T}}\right)^{-\mathbf{1}} \mathbf{H}^{\mathrm{T}}\right)\left(\mathbf{H} \mathbf{C}^{-\mathbf{1}}\right) \\
=\left(\mathbf{C}^{\mathrm{T}}\right)^{-\mathbf{1}} \mathbf{H}^{\mathrm{T}} \mathbf{H} \mathbf{C}^{-\mathbf{1}} .
\end{array}
$$

But $\mathbf{C}$ was defined through factorization hence

$$
\mathbf{H}^{\mathrm{T}} \mathbf{H}=\mathbf{C}^{\mathrm{T}} \mathbf{C} \text {. }
$$

Substitute this expression into the previous equation and obtain

$$
\begin{aligned}
\mathbf{F} \mathbf{F}^{\mathrm{T}}=\left(\left(\mathbf{C}^{\mathrm{T}}\right)^{-\mathbf{1}} \mathbf{H}^{\mathrm{T}}\right)\left(\left(\mathbf{C}^{\mathrm{T}}\right)^{-\mathbf{1}} \mathbf{H}^{\mathrm{T}}\right)^{\mathrm{T}} & =\left(\mathbf{C}^{\mathrm{T}}\right)^{-\mathbf{1}} \mathbf{H}^{\mathrm{T}} \mathbf{H} \mathbf{C}^{-\mathbf{1}} \\
& =\left(\mathbf{C}^{\mathrm{T}}\right)^{-\mathbf{1}} \mathbf{C}^{\mathrm{T}} \mathbf{C} \mathbf{C}^{-\mathbf{1}} \\
& =\mathbf{I} .
\end{aligned}
$$

Hence the transformation matrix $\mathbf{F}$ is unitary.

Theorem 2. $\mathbf{F}$ is an all pass filter.

Proof. Denote a random white noise sequence as a column vector $\mathbf{n}$, with a noise covariance matrix given by $\mathbf{I}$ the identity matrix. Write the noise covariance matrix of filtered noise $\mathbf{n}_{\mathbf{f}}=\mathbf{F n}$ as

$$
\boldsymbol{\Sigma}=E\left\{\mathbf{n}_{\mathbf{f}} \mathbf{n}_{\mathbf{f}}^{\mathrm{T}}\right\}
$$

It follows that

$$
\boldsymbol{\Sigma}=E\left\{\mathbf{F n}(\mathbf{F n})^{\mathrm{T}}\right\}=E\left\{\mathbf{F n n}{ }^{\mathrm{T}} \mathbf{F}^{\mathrm{T}}\right\}=\mathbf{F} E\left\{\mathbf{n} \mathbf{n}^{\mathrm{T}}\right\} \mathbf{F}^{\mathrm{T}} .
$$

But since $\mathbf{n}$ is white noise, $\mathbf{E}\left\{\mathbf{n} \mathbf{n}^{\mathrm{T}}\right\}=\mathbf{I}$, and hence

$$
\boldsymbol{\Sigma}=\mathbf{F F}^{\mathrm{T}} \text {. }
$$

However $\mathbf{F}$ is unitary, thus it follows that

$$
\boldsymbol{\Sigma}=E\left\{\mathbf{n}_{\mathbf{f}} \mathbf{n}_{\mathbf{f}}^{\mathrm{T}}\right\}=\mathbf{F} \mathbf{F}^{\mathrm{T}}=\mathbf{I} .
$$

Hence the filtered noise $\mathbf{n}_{\mathbf{f}}=\mathbf{F n}$ has a white power spectral density, and $\mathbf{F}$ is an all-pass filter. 